\title{
Décentralisation et ruptures méthodologiques
}

Le cas de l'Argentine

Decentralisation and fragmentation in teaching methodology - the Argentine -

experience

Descentralización y ruptura metodológica. El caso argentino

Mariano Palamidessi et Daniel Galarza

\section{(2) OpenEdition}

Journals

Édition électronique

URL : https://journals.openedition.org/ries/1894

DOI : 10.4000/ries. 1894

ISSN : 2261-4265

Éditeur

France Education international

Édition imprimée

Date de publication : 1 avril 2002

Pagination : 101-114

ISBN : 2-84520-553-8

ISSN : $1254-4590$

Référence électronique

Mariano Palamidessi et Daniel Galarza, « Décentralisation et ruptures méthodologiques », Revue internationale d'éducation de Sèvres [En ligne], 29 | avril 2002, mis en ligne le 25 novembre 2011 , consulté le 07 juillet 2021. URL : http://journals.openedition.org/ries/1894 ; DOI : https://doi.org/ 10.4000/ries. 1894 


\section{Décentralisation et ruptures méthodologiques}

\section{Le cas de l'Argentine}

\section{Mariano Palamidessi et Daniel Galarza}

Quelques éléments d'analyse sur l'expérience vécue par les élèves dans les écoles primaires argentines ${ }^{1}$ sont développés dans cet article. L'étude définit l'environnement contextuel, les pratiques d'enseignement et les activités d'apprentissage avant d'énoncer quelques hypothèses sur les relations qui existent entre les élèves et le savoir dans les écoles de notre pays.

Cette étude se divise en trois parties. Dans un premier temps, nous situons les contextes matériels dans lesquels se déroule le travail scolaire et définissons le profil type des élèves argentins. Nous présentons ensuite, après avoir étudié des cahiers d'école et avoir analysé certaines données statistiques, les activités qui ont lieu dans les classes et décrivons les méthodes d'évaluation des élèves. Enfin, nous faisons quelques observations en guise de conclusion.

\section{UN ENVIRONNEMENT HÉTÉROGÈNE ET COMPLEXE}

Il est difficile de présenter un environnement représentatif des contextes et des pratiques pédagogiques dans lequel se déroulent les activités scolaires des écoles primaires de notre pays pour diverses raisons. Nous retiendrons principalement trois d'entre elles.

\section{Le caractère fédéral et les faibles niveaux de coordination et d'intégration du système éducatif}

$\mathrm{Au}$ cours des deux dernières décennies du XIX $\mathrm{X}^{\mathrm{e}}$ siècle, le système éducatif argentin s'est organisé dans un contexte centralisateur (Paviglianiti,

\footnotetext{
1. Il convient d'apporter des éclaircissements préliminaires :

1) conformément à la Loi fédérale d'éducation de 1993 (Ley Federal de Educación), l'éducation générale basique (Educación General Básica (EGB)) de la République d’Argentine dure neuf ans et est obligatoire. Elle se divise en trois cycles de trois ans : le premier cycle de la $1^{\text {re }}$ à la $3^{e}$ année, le deuxième cycle de la $4^{\mathrm{e}}$ à la $6^{\mathrm{e}}$ année et le troisième cycle de la $7^{\mathrm{e}}$ à la $9^{\mathrm{e}}$ année. Comme il s'agit d'une structure en transition, cet article s'intéresse à l'ancien système primaire (de la $1^{\text {re }}$ à la $7^{\mathrm{e}}$ année), modèle institutionnel qui constitue encore l'enseignement obligatoire. Ainsi les données traitées portent sur les deux premiers cycles de l'EGB;

2) il faut avoir à l'esprit que l'enseignement privé n'est pas un secteur homogène, il regroupe des institutions qui accueillent des élèves de classes sociales moyennes ou supérieures et des écoles confessionnelles financées par l’État pour les enfants des catégories sociales défavorisés.
} 
1989). Au XX siècle, deux réseaux d'écoles primaires ont cohabité en Argentine : le réseau national et le réseau provincial ${ }^{2}$. Le Conseil national (et les autorités provinciales) définissaient les normes, déterminaient les contenus et les méthodes d'enseignement, formaient et désignaient les enseignants, géraient et contrôlaient l'enseignement et les livres scolaires. Vers la fin des années 1960, l'hégémonie du modèle centralisé commença à être remise en question. "L'État éducateur» s'est ébranlé pour les raisons suivantes (Márquez, 1995 et Fernández, Lemos et Wiñar, 1997) : diverses fonctions ont été déléguées et transférées aux juridictions ${ }^{3}$; le secteur privé s'est accru à partir des années 1970 (plus de $20 \%$ d'inscriptions au niveau du primaire) et la capacité de contrôle et d'inspection des écoles a connu une diminution progressive. Ce processus de fédéralisation du système et la décentralisation des moyens attribués au secteur ${ }^{4}$ sont survenus en même temps. En bref, il est difficile de donner une définition du cadre scolaire car l'organisation est très disparate, complexe et mal coordonnée.

\section{Un pays frappé par des différences sociales et régionales croissantes}

Une croissance du chômage et du sous-emploi et une chute des recettes des classes moyennes et défavorisées accompagnent le processus décentralisateur. Les inégalités sociales et les différences qui existent entre les régions intégrées et les régions marginalisées ${ }^{5}$ accentuent ces évolutions. La détérioration des salaires et du statut professionnel des enseignants est liée à ce processus.

\section{Une pénurie d'études systématiques}

Bien que la production académique locale en éducation ait augmenté et se soit diversifiée au cours des deux dernières décennies, nous ne disposons d'aucune description empirique à caractère systématique sur les pratiques de

2. D’après la Constitution nationale de 1853 , l'enseignement primaire est sous la tutelle des États provinciaux. Confrontés à un manque de ressources, la plupart ont sollicité l'aide du gouvernement. En 1905, le Congrès de la nation a donné son aval pour que des écoles primaires nationales soient créées dans les provinces.

3. Au cours des deux dernières décennies, les écoles primaires et secondaires nationales ont été mises sous la responsabilité des provinces. La formation des enseignants a également connu un transfert. Alors qu'elle se faisait auparavant dans le secondaire, elle se ferait alors au niveau de l'enseignement supérieur non universitaire et elle serait ouverte au secteur privé. Ce changement a fortement bouleversé l'ordre établi par les écoles normales (Escuelas Normales) qui assuraient un programme pédagogique commun au niveau national.

4. Il existe aujourd'hui une forte inégalité dans les dépenses par élève au niveau primaire entre les différentes juridictions, de 700 à 2.500 \$ US par élève/an (Cosse, 2001).

5. Les différentes régions sont les suivantes: Metropolitana (ville de Buenos Aires et Gran Buenos Aires); Pampeana (l'autre partie de la Province de Buenos Aires, Córdoba, Entre Ríos, La Pampa et Santa Fe); Patagonia (Chubut, Neuquén, Río Negro, Santa Cruz et Tierra del Fuego) ; Cuyo (Mendoza, San Juan et San Luís) ; Nord-Ouest (Catamarca, Jujuy, La Rioja, Salta, Santiago del Estero et Tucumán) et Nord-Est (Corrientes, Chaco, Formosa et Misiones). 
l'enseignement et les activités scolaires. L'analyse de revues spécialisées et de congrès sur l'éducation au cours des cinq dernières années dévoile une pénurie presque totale de travaux sur ces sujets ${ }^{6}$. Les études traitent ces sujets au niveau local et les données sont difficilement comparables. Cet article a été rédigé essentiellement à partir de données statistiques fournies par le ministère et de certains travaux et rapports d'ordre qualitatif.

\section{LES CONTEXTES MATÉRIELS DE L'APPRENTISSAGE}

Un bref rappel des données concernant les établissements équipés de bibliothèques, de salles informatiques ou de laboratoires montre les différences de contextes matériels dans lesquels étudient les élèves argentins. L’infrastructure des écoles primaires varie en fonction des provinces et du public qu'elles accueillent.

Tableau no 1

Établissements scolaires qui ont des espaces pédagogiques différenciés

(en pourcentage, par juridiction choisie)

\begin{tabular}{|l|c|c|c|c|c|}
\hline Province & $\begin{array}{r}\text { Salle } \\
\text { informatique }\end{array}$ & $\begin{array}{c}\text { Bibliothèque } \\
\text { d'arts } \\
\text { plastiques }\end{array}$ & Salle & Laboratoire & $\begin{array}{c}\text { Salle de } \\
\text { musique }\end{array}$ \\
\hline Ciudad de Bs. Aires (Metropolitana) & 68,65 & 67,75 & 35,64 & 39,45 & 46,51 \\
\hline Córdoba (Pampeana) & 24,89 & 27,53 & 9,01 & 14,19 & 10,55 \\
\hline Misiones (Nord-Est) & 11,85 & 28,93 & 3,95 & 7,99 & 4,68 \\
\hline Santiago del Estero (Nord-Ouest) & 4,42 & 12,31 & 2,31 & 3,54 & 3,67 \\
\hline Tierra del Fuego (Patagonia) & 44,87 & 73,08 & 23,08 & 25,64 & 29,49 \\
\hline Total & 26,06 & 31,73 & 9,67 & 15,72 & 13,15 \\
\hline
\end{tabular}

Source : Recensement national d'infrastructure éducative de 1998 (Censo Nacional de Infraestructura EducativaCENIE). Programme national de promotion de la qualité éducative (Programa Nacional de Promoción de la Calidad Educativa). Réseau fédéral d'information éducative (Red Federal de Información Educativa). Choix des catégories et juridictions.

Un tiers des bâtiments scolaires dispose d'une bibliothèque et seulement un sur quatre d'une salle informatique ${ }^{7}$. Les différences régionales sont frappantes : tandis qu'à Tierra del Fuego 7 établissements scolaires sur 10 ont

6. Nous faisons référence aux articles publiés dans la Revista Argentina de Educación (Revue argentine d'éducation), la Revista del Instituto de Investigaciones en Ciencias de la Educación (Revue de l'Institut de recherches en sciences de l'éducation) de la Faculté de Philosophie et Lettres de l'Université de Buenos Aires, et Propuesta Educativa (Proposition éducative) de la Faculté latino-américaine de sciences sociales. Les congrès les plus pertinents ont été organisés par l'Université de Buenos Aires (1996 et 2000) et l'Université nationale de Comahue (2000 et 2001).

7. Dans une même enceinte scolaire peuvent cohabiter plusieurs établissements scolaires de différents niveaux. La plupart des bâtiments abritent des établissement de niveau primaire. 
une bibliothèque, à Santiago del Estero il n'y en a que $12 \%$. Le même schéma se reproduit lorsqu'il s'agit d'autres espaces scolaires, comme les salles d'arts plastiques ou les laboratoires. Les écoles du Nord-Ouest et du Nord-Est du pays sont encore moins bien équipées ${ }^{8}$.

En 1998, seulement $39 \%$ des écoles primaires argentines possédaient au moins un ordinateur pour les élèves. La quantité et les caractéristiques techniques de cet équipement varient considérablement en fonction de la province et du secteur, les écoles privées et deux provinces (Metropolitana et Patagonia) disposant de plus d'équipement et de meilleure qualité. L'accès à Internet n'est pas encore très répandu : seulement $4 \%$ des écoles primaires pouvaient se connecter, $19 \%$ pour le secteur privé (Galarza et Gruschesky, 2001). Des études en cours montrent que seulement $30 \%$ des écoles qui accueillent des enfants dont les parents ont un faible niveau scolaire (niveau primaire et moindre) possédaient du matériel informatique, contre $87 \%$ des écoles qui accueillent des enfants dont les parents ont un niveau scolaire élevé. $30 \%$ des écoles équipées d'ordinateurs disposaient de logiciels éducatifs pour l'enseignement de la langue espagnole et des mathématiques, mais il faut préciser que les pourcentages sont deux fois plus élevés dans le secteur privé que dans le secteur public ${ }^{9}$.

\section{QUI SONT LES ÉLÈVES ARGENTINS ?}

Dans un pays aux inégalités croissantes en termes socio-économiques, la diversité des conditions de vie des élèves est un autre élément majeur qu'il faut prendre en compte.

D'après l'enquête nationale d'évaluation de la qualité de 1999 (Operativo Nacional de Evaluación de la Calidad), presque $25 \%$ des directeurs d'écoles primaires estimaient que peu d'élèves étaient «bien nourris ». $30 \%$ des directeurs des écoles publiques affirmaient que seulement « un peu plus de la moitié » des élèves allaient régulièrement à l'école. En revanche, $90 \%$ des directeurs d'écoles privées déclaraient que "presque tous » les élèves assistaient régulièrement aux cours. Dans certaines provinces (Patagonia, Metropolitana et Pampeana) le pourcentage de présence en cours est supérieur à la moyenne nationale, ce pourcentage est nettement en dessous dans d'autres provinces (Cuyo, Nord-Est et Nord-Ouest). ${ }^{10}$

8. On ne dispose d'aucune donnée fiable concernant le matériel pédagogique dont disposent les écoles, ni sur la quantité de livres disponibles dans les bibliothèques, ni même sur la réactualisation de ces livres.

9. Il s'agit d'une étude fondée sur des questionnaires distribués aux directeurs dans le cadre de l'enquête d'évaluation de la qualité (Operativo de Evaluación de la Calidad) qui se fait sous la direction de l'Unité de recherches éducatives du ministère de l'Éducation de la nation. À partir de ces données, on peut estimer que plus de $50 \%$ des écoles primaires possèdent au moins un ordinateur.

10. Pour comprendre ces informations, il faut savoir que beaucoup d'écoles publiques remplissent une fonction d'assistance importante. Elles distribuent des repas aux élèves, ce qui contribue à promouvoir l'assistance scolaire. 
En général, les directeurs et les enseignants des écoles publiques se retrouvent face à des élèves désavantagés au départ par rapport à ceux qui sont inscrits dans des écoles privées. Plus de $70 \%$ des directeurs d'écoles privées précisent que "plus de la moitié » ou "presque tous » leurs élèves ont des acquis alors qu'un peu plus de $33 \%$ des directeurs des écoles publiques déclarent la même chose. $60 \%$ des directeurs d'écoles privées considèrent que "presque tous» leurs élèves sont aidés par leurs parents alors que $62 \%$ des directeurs d'écoles publiques estiment que "très peu » ou « moins de la moitié » de leurs élèves bénéficient d'une aide à la maison.

Cette diversité de conditions de vie n'affecte pas uniquement les élèves. Des études récentes détaillent avec précision dans quelle mesure ces différences sociales et régionales ont également des répercussions sur les enseignants ${ }^{11}$.

\section{LES ÉLÈVES ET LES MANUELS SCOLAIRES}

En Argentine, les manuels scolaires sont rédigés et édités par le secteur privé. Depuis l'avènement de l'ordre constitutionnel (1983), les manuels scolaires ne sont soumis ni à un accord officiel, ni à des inspections techniques et pédagogiques. Au cours de la dernière décennie, l'État national a relancé une politique de distribution de manuels et de matériel scolaires pour les élèves les plus défavorisés (Gluz, 2001). Il s'agit cependant d'une politique d'ordre compensatoire qui ne concerne qu'une partie des élèves. Le matériel et les manuels sont payés par les familles. Certaines études mettent en évidence les difficultés auxquelles est confrontée une grande partie de la population pour payer les dépenses en matériel, manuels et cahiers. Comme le souligne Tenti, « il est très probable que cette inégale disponibilité des ressources scolaires stratégiques détermine en partie le genre de stratégie pédagogique et la qualité des apprentissages effectivement développés» (1992: 71).

On ne dispose d'aucun chiffre fiable, il est cependant évident que l'utilisation de manuels scolaires par élève et par an a diminué au cours de la dernière décennie. Plus de la moitié des enseignants des écoles publiques interrogés lors d'une enquête ont avoué qu'ils ne recommandaient aucun manuel scolaire à leurs élèves. La demande de manuels par les enseignants semble être étroitement liée aux niveaux de rendement académique des différentes écoles : $73 \%$, dans les écoles à faible rendement, $50 \%$ dans les écoles à rendement moyen et $45 \%$ dans celles à haut rendement (MCyE, 1999a). On utilise essen-

11. Une étude récente révèle que plus de $10 \%$ des instituteurs dans les villes d'Argentine vivent en dessous du seuil de pauvreté. Dans le Nord-Ouest, une des provinces les plus pauvres du pays, un instituteur sur trois est pauvre. Il semblerait que plus les instituteurs sont jeunes, plus le rapport est élevé. On peut en déduire que le métier d’instituteur intéresse de plus en plus une partie de la population à faible ressources (Institut international de planification de l'éducation-instituto internacional de planeamiento de la educación, 2000). Comme élément de comparaison, on estime qu'entre $25 \%$ et $30 \%$ des familles urbaines en Argentine sont pauvres. 
tiellement les manuels scolaires pour faire des exercices et, dans une moindre mesure, pour structurer les activités ou pour rechercher des informations.

D'après une autre source, le pourcentage d'enseignants qui demandent à leurs élèves des manuels spécifiques de langue espagnole et de mathématiques sont les suivants. ${ }^{12}$

Tableau $\mathrm{n}^{\circ} 2$

Enseignants qui demandent des manuels spécifiques

(en pourcentage)

\begin{tabular}{|l|c|c|c|c|}
\hline & \multicolumn{2}{|c|}{ Langue espagnole } & \multicolumn{2}{c|}{ Mathématiques } \\
\hline & Public & Privé & Public & Privé \\
\hline $3^{\mathrm{e}}$ année du primaire & 40,2 & 45,1 & 29,2 & 41,3 \\
\hline $6^{\mathrm{e}}$ année du primaire & 39,5 & 50,7 & 39,5 & 49,2 \\
\hline
\end{tabular}

Source : tableau établi en fonction des données recueillies sur les questionnaires distribués à des enseignants en 1999 lors de l'enquête nationale d'évaluation de la qualité.

Ces chiffres mettent en évidence les conditions matérielles et symboliques auxquelles doivent s'adapter les enseignants et les élèves. Cependant, certains auteurs affirment que cette pénurie de manuels dans la vie scolaire n'est pas uniquement due aux difficultés économiques ; elle serait également liée au fonctionnement d'une "idéologie pédagogique dominante » qui rejette de plus en plus le manuel scolaire car il est « insuffisant, unilatéral, dépassé » et privilégie " le quotidien ", " l'apprentissage par le jeu » et « le travail en équipe avec divers matériels» (Verón, 1999 : 132). Un grand nombre d'enseignants considèrent que le manuel scolaire est malheureusement associé au caractère encyclopédique rébarbatif du manuel traditionnel et va à l'encontre des processus de construction du savoir. ${ }^{13}$

12. La différence entre les données provenant de deux sources différentes est justifiée étant donné que la recherche publiée par le ministère en 1999 (MCyE, 1999b) a été réalisée sur la base d'un échantillon représentatif de 72 écoles sélectionnées en fonction des résultats (bas, moyens et élevés) obtenus lors de l'enquête d'évaluation de la qualité de 1997 (Operativo de Evaluación de la Calidad). Les écoles qui obtiennent de meilleurs résultats sont plus nombreuses, ce sont en majorité des écoles qui accueillent des élèves économiquement privilégiés.

13. «Associé à la pédagogie traditionnelle, le manuel est dépassé et obsolète. L'ordre, la structuration et le caractère systématique du manuel le déprécient (...) Une idéologie pédagogique qui dévalorise le manuel, aussi bien par ses contenus limités que par son format périmé. Un bon instituteur est un instituteur autosuffisant qui rejette le manuel scolaire » (Verón, 1999:128). « Cependant, en début d’année il se peut que des parents et des élèves demandent à l'enseignant de leur recommander un manuel, pour l'enseignant c'est une "demande de sécurité" : les parents posent cette question pour pouvoir aider leurs enfants ou contrôler les devoirs ; les élèves posent cette question afin de pouvoir se tourner vers une source de connaissances basiques structurée en cas de besoin. Les enseignants ont tendance à refuser de donner une réponse, soit parce que le manuel est inutile, soit parce qu'ils l'utilisent d'une manière particulière, parfois honteuse (...). L'enseignant préfère que la “demande de sécurité" repose sur lui et non sur le manuel. Il est à la recherche d'une revalorisation sociale de son rôle pédagogique (idem: 129) ». 


\section{LES ACTIVITÉS DES ÉLÈVES D'APRÈS LES CAHIERS : LA DURABILITÉ D'UN PROGRAMME COMMUN ?}

Les cahiers et les classeurs sont une des meilleures sources disponibles pour analyser les activités des élèves dans les écoles primaires argentines ${ }^{14}$. Un rapport réalisé par le ministère de l'Éducation de la nation (1999b) sur des cahiers de tout le pays ${ }^{15}$ révèle le temps accordé à chaque matière du programme et le type d'activités proposées à l'école primaire. Au cours des deux premiers cycles du primaire, on consacre plus de temps à l'enseignement de la langue espagnole et des mathématiques. Bien entendu ces enseignements prennent plus de temps pendant le premier cycle, les efforts se concentrent alors sur la lecture, l'écriture et le calcul. Au cours du deuxième cycle, on accorde plus de temps à l'étude des sciences sociales, et dans une moindre mesure, des sciences naturelles. Cependant, les activités liées aux mathématiques et à la langue espagnole occupent les élèves une grande partie de la journée.

Tableau no 3

Leçons par matière dans certaines classes du premier et du deuxième cycles

\begin{tabular}{|l|c|c|c|c|}
\hline \multirow{2}{*}{ Matières } & \multicolumn{2}{|c|}{ Premier cycle } & \multicolumn{2}{c|}{ Deuxième cycle } \\
\hline & No de leçons & $\%$ & No de leçons & $\%$ \\
\hline Langue espagnole & 1004 & 44,5 & 801 & 32,0 \\
\hline Mathématiques & 899 & 39,9 & 911 & 36,4 \\
\hline Sciences sociales & 264 & 11,7 & 425 & 17,0 \\
\hline Sciences naturelles & 89 & 3,9 & 367 & 14,6 \\
\hline Total & 2256 & 100 & 2504 & 100 \\
\hline
\end{tabular}

Source : ministère de la Culture et de l’Éducation (1999b).

L'analyse des cahiers met en évidence la durabilité de nombreux contenus et de méthodes typiques de l'instruction élémentaire. Il s'agit d'un " curriculum mosaïque ( Feldman, 1993), qui résulte des modifications (ajouts et suppressions) peu à peu apportées par les instituteurs et les écoles. Mais, dans quelle mesure la hausse des inégalités sociales et économiques et la décentralisation du système éducatif qui ont eu lieu au cours des dernières décennies ontelles des répercussions sur cette configuration? Dans quelle mesure les

14. Dans une étude qui analyse l'histoire des cahiers et leur évolution tout au long de la deuxième moitié du XXe siècle, Gvirtz (1997) signale que «l'activité » est l'axe qui articule les cahiers d'école.

15. Le rapport a été rédigé à partir des cahiers d'élèves du premier cycle de l'école primaire ( $1^{\text {re }}$ et $2^{\mathrm{e}}$ années) et du deuxième cycle ( $6^{\mathrm{e}}$ année) dans les 24 juridictions qui divisent le pays. 


\section{Langue espagnole}

Dans le premier cycle, lorsqu'il s'agit d'alphabétiser, les activités portent sur l'aprestamiento ${ }^{16}$, la dictée de mots et de phrases pour que les élèves écrivent ; les routines, qui marquent la dimension régulatrice de la langue écrite (mettre dans des endroits précis son nom, la date, le nom de la maîtresse), la production de mots à partir de lettres ou de syllabes et diverses formes de recopiage. Lorsqu'il s'agit de privilégier la lecture et la compréhension, on travaille l'appariement (l'association d'un dessin et d'un mot ou son écriture) ; les activités de discrimination phonique (par exemple, séparer en syllabes un mot) ; celles de discrimination visuelle (reconnaître les lettres d'un mot ou les mots d'un dessin) et celles de monitoring de la compréhension de lecture (réaliser une illustration d'après une consigne donnée à la suite de la lecture d'un texte).

Dans le deuxième cycle, plus de la moitié des leçons correspondent à des activités en rapport avec la morphologie, la syntaxe, la sémantique, l'orthographe et la ponctuation. Dans une moindre mesure, on propose des leçons de littérature, celles qui abordent des notions de communication et différents types de textes. Lors des activités consacrées à la lecture, on demande souvent de lire et de répondre à quelques questions, de résumer, d'identifier différents types de textes et de chercher des tournures grammaticales ou normatives. Les questions semblent être le moyen le plus utilisé pour contrôler la compréhension de lecture. On accorde moins d'importance aux activités de lecture de textes littéraires, essentiellement des récits courts et de la poésie. D'autre part, les activités d'écriture sont liées au développement de différents types de genres discursifs comme les narrations, les résumés, les différents types de lettres (formelle, familière), les dialogues et les publicités.

\section{Mathématiques}

Dans le premier cycle, $90 \%$ des leçons sont consacrées à l'enseignement des nombres et des opérations. Les activités concernant la géométrie ou les mesures sont très peu nombreuses. Au cours des premières années, il est très fréquent de distribuer des photocopies afin de faciliter la réalisation des tâches. De même, il est courant d'avoir recours à des matériels concrets (pour la manipulation matérielle ou les représentations graphiques) pour travailler sur les nombres. Parmi les activités réalisées le plus souvent par les élèves on peut citer : mettre des nombres dans l'ordre, les écrire et résoudre des opérations de calcul par écrit. Les problèmes d'application sont très nombreux : lire un énoncé avec des données et utiliser ces données pour réaliser une addition sur la base d'une procédure unique.

Dans le deuxième cycle, les sujets les plus abordés sont les nombres et les opérations ( $3 / 4$ des registres), puis la géométrie et la mesure. Logique, ensembles et statistiques sont des thèmes moins étudiés. Les activités semblent suivre, de manière majoritaire, les étapes suivantes : définition, calcul/exemple et application. Les problèmes se caractérisent par la présence de contextes très stéréotypés. "Les nombres décimaux en rapport avec les prix et les mesures, les nombres naturels à plusieurs chiffres avec les numéros de documents ou les distances planétaires, les mesures de temps associées aux montres digitales, pour le travail avec des droites parallèles et perpendiculaires apparaissent les plans de rues » (MCyE, 1999b: 143).

16. Aprestamiento se réfère aux « activités de développement de la motricité, des capacités visuelles spatiales et de la manipulation d'instruments nécessaires pour la maîtrise de la technologie de la langue écrite » (MCyE, 1999: 41). 
L'utilisation de ressources auxiliaires se limite à l'usage des calculatrices. $82,3 \%$ des instituteurs de la $6^{\mathrm{e}}$ année dans les écoles publiques et 95,7\% dans les écoles privées demandent des outils de géométrie ${ }^{17}$. Dans les cahiers, on ne fait que très rarement référence à l'utilisation de manuels spécifiques dans ce domaine. Il n'y a pas beaucoup de corrections sur les cahiers. Les activités sont souvent corrigées longtemps après leur réalisation.

\section{Sciences sociales}

Dans les cahiers des élèves, les activités consacrées à cette matière apparaissent nettement divisées entre deux thèmes : la géographie et l'histoire. La géographie de type descriptif est la plus enseignée, c'est-à-dire la géographie physique (territoire, relief, hydrographie, biomasse) et la géographie politique (la division politique du territoire). Quant à l'histoire, «on perçoit une logique de sélection fondée sur la combinaison d'une date marquant un événement important associée au récit historique qui l'accompagne, l'histoire enseignée débute avec les civilisations précolombiennes et en général ne dépasse pas la période de la révolution et des guerres d'indépendance. Une vision de l'histoire politique et militaire est très présente dans les contenus ; les acteurs sont toujours d'illustres personnages " (MCyE,1999b: 206). On retrouve souvent dans les cahiers une leçon de géographie, suivie de la leçon d'histoire qui correspond, leçons entrecoupées d'activités liées à la date du jour. En ce qui concerne la géographie, la problématique environnementale ou l'utilisation de ressources naturelles ne font pas partie des programmes de géographie physique et politique.

D'un point de vue méthodologique, en sciences sociales, on consacre du temps à l'élaboration des axes chronologiques et aux questionnaires de réponse individuelle, afin d'évaluer l'acquisition des connaissances transmises par l'enseignant. Les élèves doivent souvent chercher et noter sur leurs cahiers une information ponctuelle qu'ils ont recherchée sur des supports écrits (manuels, dictionnaires, revues). Parfois, les élèves travaillent sur des activités de conceptualisation et d'organisation de l'information. Ils doivent faire des tableaux, des schémas ou des diagrammes. Quelquefois, on leur demande d'interpréter des textes ou on leur pose des questions qui requièrent un certain type d'analyse ou de recontextualisation de l'information.

\section{Sciences naturelles}

Dans le domaine des sciences naturelles, la biologie est très présente (plus de la moitié des leçons enregistrées), puis l'astronomie, la chimie, la géologie et la physique (toutes autour de $10 \%$ ). Les activités liées à l'information verbale sont les plus nombreuses : recopier des données, répondre à des questionnaires ; reconnaître des faits, des définitions de concepts et des relations entre les concepts. Quelques activités sont consacrées à la production de synthèses conceptuelles. Peu d'activités permettent aux élèves d'observer, d'interpréter des évidences à l'aide de dessins fournis pour la recherche scolaire. Lorsque l'activité met l'accent sur les procédés d'investigation, on remarque une prédominance du traitement d'exposition de la part de l'enseignant et l'exécution reproductive de la part des élèves.

17. Données déduites en fonction des questionnaires soumis à des enseignants dans le cadre de l'enquête nationale de l'évaluation de la qualité de 1999 (Operativo Nacional de Evaluación de la Calidad). 
orientations pédagogiques officielles qui encouragent une plus grande autonomie et réflexivité des élèves face à la connaissance influencent-elles cette configuration?

\section{COMMENT LES ÉLÈVES SONT-ILS ÉVALUÉS?}

Les activités et les connaissances des élèves sont évaluées en fonction d'un certain nombre de critères, d'instruments et d'une périodicité propres à chaque enseignant. Chaque établissement les définit par rapport à l'évaluation. L'État détermine les règles d'accréditation et de passage au niveau supérieur et, dans les écoles publiques, les instruments utilisés pour communiquer les notes et les qualifications aux parents.

Ce tableau montre que les enseignants privilégient les formes directes et personnelles lorsqu'il s'agit d'évaluer les élèves (entrées 3 et 4 du tableau). Les devoirs à la maison sont également un des instruments de prédilection.

Tableau no 4

Formes et fréquences d'évaluation des élèves. Catégories choisies

\begin{tabular}{|l|c|c|c|c|c|c|c|c|}
\hline & \multicolumn{4}{|c|}{ Langue espagnole } & \multicolumn{3}{c|}{ Mathématiques } \\
\hline & \multicolumn{2}{|c|}{$\mathbf{3}^{\mathbf{e}}$ année } & \multicolumn{2}{|c|}{$\mathbf{6}^{\mathbf{e}}$ année } & \multicolumn{2}{c|}{$\mathbf{3}^{\mathbf{e}}$ année } & \multicolumn{2}{c|}{$\mathbf{6}^{\mathbf{e}}$ année } \\
\hline $\begin{array}{l}\text { Très } \\
\text { fréquent }\end{array}$ & $\begin{array}{c}\text { Jamais } \\
\text { ou } \\
\text { très peu }\end{array}$ & $\begin{array}{c}\text { Très } \\
\text { fréquent }\end{array}$ & $\begin{array}{c}\text { Jamais } \\
\text { ou } \\
\text { très peu }\end{array}$ & $\begin{array}{c}\text { Très } \\
\text { fréquent }\end{array}$ & $\begin{array}{c}\text { Jamais } \\
\text { ou } \\
\text { très peu }\end{array}$ & $\begin{array}{c}\text { Très } \\
\text { fréquent }\end{array}$ & $\begin{array}{c}\text { Jamais } \\
\text { ou } \\
\text { très peu }\end{array}$ \\
\hline $\begin{array}{l}\text { Tests objectifs } \\
\text { à réponses multiples }\end{array}$ & 17,6 & 15,1 & 12,9 & 13,6 & 15,5 & 13,4 & 10,9 & 13,9 \\
\hline $\begin{array}{l}\text { Tests de questions } \\
\text { ouvertes }\end{array}$ & 15,5 & 10,0 & 23,6 & 6,2 & 12,0 & 17,2 & 14,1 & 18,5 \\
\hline $\begin{array}{l}\text { Interrogations } \\
\text { aux élèves } \\
\text { individuellement }\end{array}$ & 64,0 & 2,5 & 51,7 & 2,2 & 59,7 & 2,3 & 53,5 & 2,0 \\
\hline $\begin{array}{l}\text { Observation } \\
\text { de la participation } \\
\text { des élèves en cours }\end{array}$ & 79,9 & 0,0 & 81,2 & 0,3 & 79,6 & 0,2 & 80,0 & 0,0 \\
\hline Devoirs & 40,2 & 7,5 & 30,7 & 7,0 & 41,8 & 5,4 & 31,4 & 6,4 \\
\hline $\begin{array}{l}\text { Travail de recherche } \\
\text { en groupe }\end{array}$ & 14,2 & 10,9 & 33,1 & 4,1 & 11,1 & 14,4 & 24,7 & 8,5 \\
\hline $\begin{array}{l}\text { Travail de recherche } \\
\text { individuelle }\end{array}$ & 16,3 & 13,0 & 17,5 & 8,1 & 12,8 & 12,9 & 13,2 & 14,0 \\
\hline
\end{tabular}

Source : tableau élaboré à partir de données recueillies dans les questionnaires complémentaires distribués aux enseignants pour l'enquête nationale d'évaluation de la qualité de 1999 (Operativo Nacional de Evaluación de la Calidad). 
L'évaluation des recherches, en particulier celles qui sont réalisées en groupe, est plus importante dans les classes de niveau supérieur. Cependant, le concept scolaire de " recherche " semble être associé à la recherche d'information dans les livres, les revues et, dans certaines écoles, dans les encyclopédies sur CD Rom. Les interrogations écrites en tous genres sont moins courantes ; elles sont plus fréquentes dans les classes de niveaux supérieurs et dans le secteur privé.

\section{ÉVolutions ACtuelles}

Les pratiques de l'enseignement et les activités des élèves ont été déterminées historiquement, elles se transforment pour devenir le résultat de processus sociaux, économiques et culturels complexes qui bouleversent et transcendent les efforts officiels de réforme (Goodson, 1995). L'expérience scolaire, l'autonomie et la relation entre les élèves et le savoir se construisent pour former une structure où cohabitent le passé et le présent du système scolaire.

Il y a vingt ans déjà, Juan Carlos Tedesco (1983:70) signalait que : " il est nécessaire de préciser que si la détérioration est un aspect général qui affecte le système éducatif argentin dans son ensemble, une de ses expressions les plus significatives est la perte de l'homogénéité qualitative qui l'a caractérisé historiquement ». ${ }^{18}$ Cependant, il est encore possible de remarquer dans l'école primaire la durabilité d'un programme typique de contenus et d'activités propre à l'instruction élémentaire de masse (Benavot, Cha, Kamens, Meyer, Wong, 1992). Les cahiers montrent qu'au cours des premières années, les activités proposées concernent encore essentiellement les rudiments de l'écriture et du calcul et insistent sur les règles de socialisation de base. Dans les classes de niveaux intermédiaire et supérieur, on introduit des leçons d'histoire et de géographie et des concepts élémentaires de biologie et de sciences naturelles. Ce programme traditionnel laisse peu de place à l'autonomie des élèves et offre peu de possibilités d'introduction de la diversité culturelle, mais il a édifié dans le temps un modèle scolaire uniforme pour tout le pays.

$\mathrm{Au}$ cours des trois dernières décennies, l'école primaire a connu en Argentine et dans le monde entier, une évolution constante des modèles pédagogiques "centrés sur l'enfant»(Caruso et Fairstein, 1996). On reconnaît ces nouvelles tendances par exemple dans la disposition des tables pour effectuer un travail en groupe, dans l'utilisation généralisée du dessin expressif et dans les propositions d'activités peu structurées et moins dirigées. En même temps, les

18. Et il ajoute : «Cette homogénéité, bien entendu, revêt depuis toujours un caractère relatif (...) Cependant, la particularité du cas argentin est le haut niveau qualitatif du segment public-étatique pendant un siècle » (Tedesco, 1983,70). 
programmes officiels se sont orientés vers une modernisation des contenus ${ }^{19}$ et vers des formes plus flexibles et ouvertes (Feldman et Palamidessi, 1994). Le programme traditionnel a incorporé peu à peu ces éléments innovants (par juxtaposition ou ajout) (Feldman, 1993) ; ce processus est lié aux conditions présentes dans chaque contexte local.

Comme on l'a vu, les élèves en Argentine vivent des expériences différentes dans divers contextes scolaires. On observe une répartition sociale des opportunités éducatives. Les expériences scolaires se diversifient sans cesse car les écoles sont de plus en plus autonomes et les contextes sociaux très variés. Ainsi, beaucoup d'écoles - en particulier les institutions privées laïques et certaines écoles publiques qui accueillent des élèves issus de catégories sociales très favorisées - ont un programme qui regroupe de nombreuses activités très variées ${ }^{20}$. Ces établissements proposent diverses manières d'aborder un sujet de connaissances qui mettent l'accent sur le travail en groupe, l'autonomie de l'élève et leur relation complexe et systématique avec le savoir.

D'autre part, une bonne partie des écoles qui accueillent des élèves issus de classes populaires ont essayé d'introduire la flexibilité des horaires, le travail en groupe et les stratégies d'enseignement indirectes (Palamidessi, 1997). On a progressivement élaboré une pédagogie qui tient compte des centres d'intérêts des élèves, de leurs contextes culturels et des connaissances qu'ils ont déjà acquises. Souvent, ces orientations pédagogiques ont entraîné une diminution du rythme d'instruction, des exigences (de devoirs à la maison, de manuels) et des attentes des instituteurs. Cette pédagogie apparaît clairement dans un discours qui privilégie la scolarisation des élèves le plus longtemps possible et la diminution de l'échec scolaire ${ }^{21}$.

Ce contexte facilite l'apparition de circuits d'expérience distincts dans lesquels certains élèves acquièrent une multitude de connaissances, dans un environnement d'apprentissage enrichi où des activités autonomes ont été développés. La majorité des élèves reçoit une formation élémentaire ${ }^{22}$. Une des

19. Au cours de l'année 1990, le ministère de l'Éducation a encouragé un processus de modernisation et d'actualisation des contenus. Il a défini un programme scolaire - appelé Contenidos Básicos Comunes (Contenus basiques communs) - pour chaque niveau du système (maternelle, primaire... formation d'enseignants).

20. On ne dispose d'aucune étude sur le nombre d'heures passées à l'école par les élèves de différentes catégories sociales. Dans les écoles publiques, la journée scolaire dure en général 4 heures. On ne possède aucune donnée sur le temps qui est effectivement consacré à l'enseignement. En général, les écoles privées qui accueillent des élèves provenant des plus hautes catégories sociales proposent un programme plus complet qui inclut l'enseignement d'une langue étrangère, des leçons d'informatique, de technologie, de natation, des cours de sports et d'autres ateliers d'arts plastiques.

21. Il convient de préciser que pour le premier et le deuxième cycles de l'école primaire (entre 1996-1999), le taux annuel de redoublement était de $6 \%$ et le taux d'abandon de $2 \%$.

22. Cette segmentation n'est pas directement liée à une distinction entre public et privé étant donné que des écoles des deux secteurs accueillent des élèves d'origines différentes. 
études citées révèle que les élèves scolarisés dans les écoles qui atteignent les plus hauts niveaux de rendement - généralement celles qui accueillent les enfants de milieux sociaux favorisés - ont plus de possibilités de se retrouver face à des instituteurs qui connaissent les styles didactiques « cognitivistes » et qui enseignent les nouveaux contenus (MCyE, 1999a).

Si la tradition centralisatrice a marqué la genèse et l'évolution du système éducatif argentin, les réformes des trois dernières décennies ont encouragé la décentralisation du système, l'autonomie des écoles et la rupture de l'homogénéité méthodologique (Palamidessi, 2001). En même temps, les ressources matérielles des écoles, le statut des enseignants et les conditions de vie d'une bonne partie des élèves se sont progressivement détériorés ou sont entrés en conflit avec les exigences d'une pédagogie plus flexible et diverse. Une partie des écoles uniquement a obtenu des ressources et des instruments leur permettant de s'adapter aux processus de réforme et aux nouvelles exigences.

Les enseignants et les écoles primaires argentines sont très hétéroclites, leur aptitude à enseigner aux élèves une plus grande autonomie et une approche réfléchie face aux connaissances systématiques (sciences, arts, sciences humaines, technologies ou sports) est très disparate. Ces capacités sont limitées par un État éducateur (et une société) en crise et une économie de marché qui n'inclue qu'une petite partie des familles et des enfants argentins.

\section{BibliographIE}

BENAVOT A., CHA Y., KAMENS D., MEYER J and WONG S. (1992) : "Knowledge for the Masses: World Models and National Curricula, 19201986 » in MEYER J., KAMENS D. and BENAVOT A., School Knowledge for the Masses: World Models and National Primary Curricular Categories in the Twentieth Century. London. Falmer, pp. 40-62.

CARUSO M. y FAIRSTEIN G. (1996) : "Las puertas del cielo. Hipótesis acerca de la recepción de la psicogénesis y el constructivismo de raíz piagetiana en el campo pedagógico argentino (1950-1981) » in PUIGGRÓS, A. Dictaduras y utopías en la historia reciente de la educación argentina (19551983). Buenos Aires. Galerna, pp.157-220.

COSSE G. (2001) : Gasto educativo, eficiencia, eficacia y equidad en Argentina (1990-1999). Buenos Aires, Ministerio de Educación, Unidad de Investigaciones Educativas.

DUSCHATZKY S. (1999) : La escuela como frontera. Buenos Aires, Paidós.

FELDMAN D. (1993) : Curriculum, maestros y especialistas. Buenos Aires, El Quirquincho. 
FELDMAN D. y PALAMIDESSI M., (1994) : "Viejos y nuevos planes. El curriculum como texto normative» in Propuesta Educativa, 11. Buenos Aires, Miño y Dávila- FLACSO, pp. 69-73.

GALARZA D. y GRUSCHESKY M. (2001) : El equipamiento informático en el sistema educativo (1994-1998). Buenos Aires. Ministerio de Educación, Unidad de Investigaciones Educativas. http://www.inv.me.gov.ar/publicaciones.htm).

GLUZ N. (2001): Análisis de los proyectos destinados a desarrollar nuevos lineamientos de política: Equidad, consenso federal y modernización de la función pública (1993-1999). Buenos Aires. Ministerio de Educación, Unidad de Investigaciones Educativas.

GOODSON I. (1995) : Historia del currículum. La construcción social de las disciplinas escolares. Barcelona. Pomares Corredor.

GVIRTZ S. (1987) : Del curriculum prescripto al curriculum enseñado. Una mirada a los cuadernos de clase. Buenos Aires. Aique.

INSTITUTO INTERNACIONAL DE PLANEAMIENTO DE LA EDUCACIÓN (2000): Los docentes y los desafíos de la profesionalización. Buenos Aires.

MINISTERIO DE CULTURA Y EDUCACIÓN (1998): Informe de avance sobre el estado de situación de la transformación curricular e institucional. Buenos Aires. Dirección General de Investigación y Desarrollo Educativo,. MINISTERIO DE CULTURA Y EDUCACIÓN (1999a) : Estado de situación de la transformación curricular e institucional. Buenos Aires. Dirección de Investigación y Desarrollo.

MINISTERIO DE CULTURA Y EDUCACIÓN (1999b): Los contenidos enseñados en cuatro áreas curriculares a través de los cuadernos de clase. Informe preliminar. Buenos Aires. Dirección de Investigación y Desarrollo. PALAMIDESSI M. (2001) : A ordem das coisas ensináveis. Uma leitura dos planos, programas e currículos para a escola primária. Tese de Doutorado. Porto Alegre. Universidade Federal do Rio Grande do Sul.

PALAMIDESSI M. (1996) : "Los inicios de la construcción discursiva del maestro constructivista en el currículum ». En Educaçao e Realidade, 21, 2. Porto Alegre. UFRGS, Jul/Dez.

PAVIGLIANiTi N. (1989) : Diagnóstico de la Administración Central de la Educación. Buenos Aires. Ministerio de Cultura y Educación.

TEDESCO J.C.: «Elementos para una sociología del currículum en Argentina » in TEDESCO J.C., BRASLAVSKY C. y CARCIOFI R., (1983) : El proyecto educativo autoritario. Argentina. 1976-1982. Buenos Aires. Flacso/GEL.

TENTI E. (1992) : La escuela vacía. Deberes del Estado y responsabilidades de la sociedad. Buenos Aires. Losada.

VERÓN E. (1999) : Esto no es un libro. Barcelona. Gedisa. 